\title{
FDTD Calculations of the Diffraction Coefficient of Vibrating Wedges
}

\author{
Monica Madrid, Member, IEEE, Jamesina J. Simpson, Member, IEEE, and Majeed M. Hayat, Senior Member, IEEE
}

\begin{abstract}
The full-vector Maxwell's equations finite-difference time-domain (FDTD) method is employed to calculate diffraction coefficients of vibrating conducting and dielectric wedges. Two-dimensional FDTD models of right-angle wedges are constructed to include total-field scattered-field incident plane-wave source conditions as well as convolutional perfectly matched-layer boundary conditions. These models are first validated by calculating the diffraction coefficient of a stationary perfect electrical conducting (PEC) right-angle wedge for comparison to uniform geometrical theory of diffraction (UTD) analytical solutions. Next, a brute-force FDTD technique for modeling wedge vibrations is utilized to calculate the Doppler diffraction coefficients of vibrating lossless and lossy wedges.
\end{abstract}

Index Terms-Diffraction coefficient, finite-difference time domain (FDTD), vibration, uniform geometrical theory of diffraction (UTD).

\section{INTRODUCTION}

$\mathbf{S}$ TRATIS et al. [1] have employed the full-vector Maxwell's equations finite-difference time-domain (FDTD) method [2], [3] to calculate diffraction coefficients of right-angle conducting and dielectric wedges. Their work is motivated by the fact that classical theories such as the uniform geometrical theory of diffraction (UTD) can be utilized to solve diffraction coefficient problems for perfect electrical conducting (PEC) wedges, but there is no analytical solution for the diffraction coefficient of problems that involve dielectric or imperfectly conducting wedges.

We extend the FDTD modeling of Stratis et al. to calculate diffraction coefficients of vibrating conducting and dielectric wedges. From an applied perspective, this letter is in support of recently developed signal-processing algorithms designed to estimate ground-object vibrations using synthetic-aperture radar platforms [4].

Manuscript received January 03, 2011; revised February 11, 2011; accepted February 21, 2011. Date of publication March 03, 2011; date of current version March 14, 2011. This work was supported by the United States Department of Energy under Award DE-FG52-08NA28782, the National Science Foundation under Award IIS-0813747, and Sandia National Laboratories. M. Madrid was supported by a National Science Foundation Graduate Research Fellowship and a Selected Professions Fellowship from the American Association of University Women.

M. Madrid and J. J. Simpson are with the Electrical and Computer Engineering Department, University of New Mexico, Albuquerque, NM 87131 USA (e-mail: simpson@ece.unm.edu).

M. M. Hayat is with the Electrical and Computer Engineering Department, University of New Mexico, Albuquerque, NM 87131 USA, and also with the Center for High Technology Materials, University of New Mexico, Albuquerque, NM 87106, USA.

Color versions of one or more of the figures in this letter are available online at http://ieeexplore.ieee.org.

Digital Object Identifier 10.1109/LAWP.2011.2121890
We employ two-dimensional (2-D), transverse magnetic $\left(\mathrm{TM}_{z}\right)$ FDTD models containing the wedge of interest, a total-field scattered-field (TFSF) incident plane-wave source condition [3], and a convolutional perfectly matched layer (CPML) boundary condition [5] to prevent unwanted reflections from the outer grid boundaries.

A brute-force method of modeling the object vibrations is implemented. This approach requires two FDTD simulations in which the diffracted and scattered fields are computed for the same plane-wave illumination, but for different stationary scattering wedges comprising: 1) the unperturbed wedge; and 2) the same wedge under peak applied strain. This brute-force technique requires that we employ a high-resolution FDTD grid in order to resolve the vibrations of the wedge. Note that, for efficiency, the recently developed surface impedance boundary condition (SBC) [6], [7] may be employed to model object vibrations in FDTD models instead of the brute-force technique. Previously, benchmark comparisons between results of FDTD models employing SBC have shown good agreement with corresponding analytical results [6] and [7] as well as with bruteforce FDTD results [8].

This letter has application to a number of situations involving electromagnetic (EM) wave interactions with arbitrary vibrating material objects. Of particular interest is the interaction of synthetic aperture radar signals with buildings having characteristic vibration signatures due to internal operating machinery and activities.

\section{Calculation of the Diffraction CoefFicient}

\section{A. Methodology}

Stratis et al. [1] implement time-gating in their FDTD models to obtain diffracted-only signals for subsequent calculations of the diffraction coefficient. Time-gating takes advantage of the different arrival times at specific observation points of the incident, reflected, near-edge diffracted, and far-edge diffracted waveforms. As a result, only the diffracted fields from the intended edge are extracted from FDTD models in a straightforward manner. These time-gated waveforms may subsequently be used to calculate the diffracted impulse response, $h(\rho, \phi, t)$. Taking the discrete Fourier transform of the diffracted impulse response to yield $H(\rho, \phi, \omega)$ would provide, in turn, the variation of the diffracted field over the spectral range of the incident wave. The diffraction coefficient $D$ is then calculated via the following:

$$
D(\rho, \phi, \omega)=H(\rho, \phi, \omega) \sqrt{r} e^{j \beta r}
$$




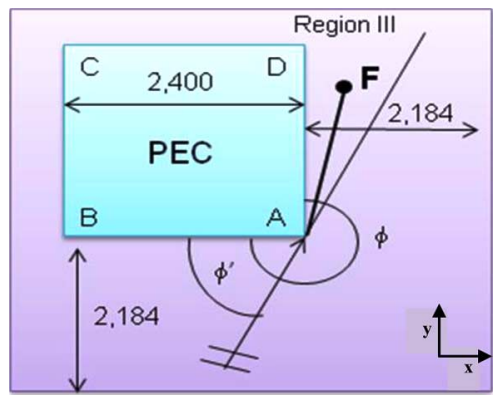

Fig. 1. Layout of the FDTD grid for computing the diffraction coefficient at Point F.

where $(\rho, \phi)$ is the observation point and $r$ is the distance from the scattering edge to the observation point.

\section{B. Model Geometry and Validation Study}

A validation study for the FDTD-calculated diffraction coefficient is performed for the case of a stationary PEC right-angle wedge. The FDTD-calculated results are compared to the analytical solutions obtained via UTD [9].

A 2-D $\mathrm{TM}_{z}$ FDTD model is generated having a total grid size of $4908 \times 4908$ cells and containing a square PEC object of dimension $2400 \times 2400$ cells. The bottom of the square PEC ( $-y$-direction side) is located 2184 grid cells from the bottom of the grid, and the right side of the square PEC is located 2184 grid cells from the right side of the grid. The incident plane-wave source is a Gaussian modulating a sinusoid at a center frequency of $850 \mathrm{MHz}$ and having a 1.3-ns duration (full width at half-maximum). The angle of incidence is $80^{\circ}$. The grid cell increment in the $x$ - and $y$-directions is set to $2.9 \mathrm{~mm}$. High resolution is chosen so that the small-amplitude vibrations considered in Section III may be resolved by this same grid.

The model is run for a total of 11039 time-steps, and the time-step increment is set to $6.8 \mathrm{ps}$ (Courant limit [3]). An observation point, denoted by Point $\mathrm{F}$ in Fig. 1, is chosen to be located slightly to the right of the square PEC at $\rho=6.3$ and $\phi=262.37^{\circ}$, in the diffracted-field-only region (Region III). The edge yielding the diffracted waves considered in this letter is located at the bottom right of the square PEC (here, a $90^{\circ}$ wedge angle), edge A. This arrangement is analogous to the wedge scenario of [1].

In order to obtain the diffraction coefficient, the Fourier transform is taken of the electric field time-waveform at the observation point $\mathrm{F}$ and normalized relative to the incident plane-wave source

$$
H(\rho, \phi, \omega)=\frac{F\left\{X_{\text {dif }}(\rho, \phi, t)\right\}}{F\left\{X_{\text {inc }}(0, \phi, t)\right\}}
$$

where the subscript "dif" denotes the diffracted field values at Point F and the subscript "inc" denotes the incident field values.

The diffraction coefficient is then computed via (1). Note that Point $\mathrm{F}$ is in the shadow scattering region, which is of practical interest for diffraction problems.

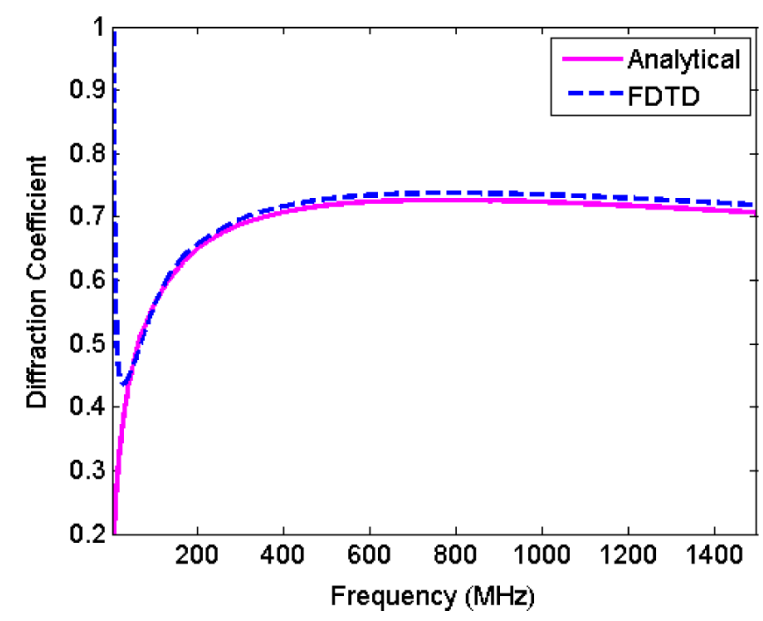

Fig. 2. Comparison between the analytical UTD solution and the FDTD results for the diffraction coefficient for right-angle PEC wedge at Point F ( $\rho=$ $\left.6.3, \phi=262.37^{\circ}\right)$.

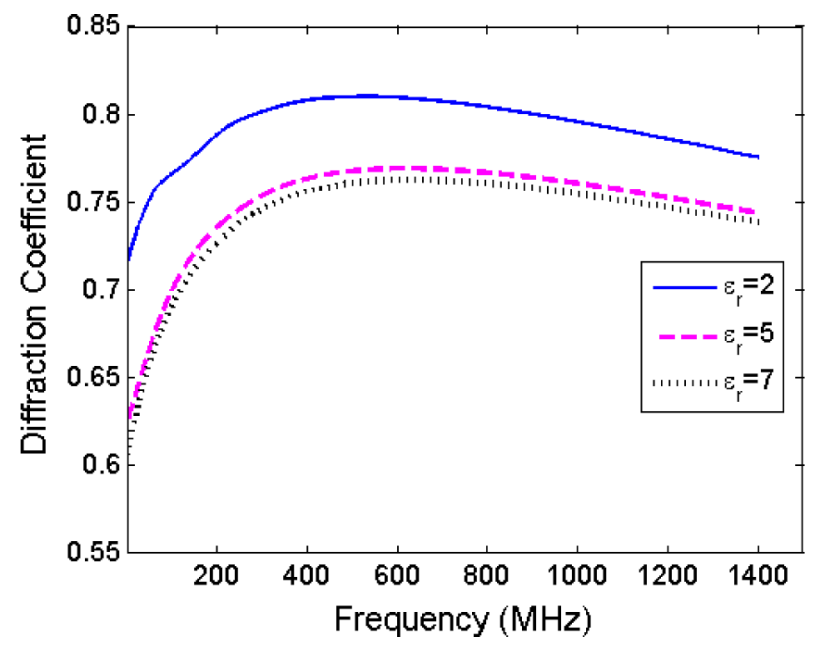

Fig. 3. Comparison between the diffraction coefficients for lossless, unperturbed dielectric right-angle wedges having a relative permittivity of 2,5 , and 7 , respectively.

To obtain the UTD analytical solution for comparison to the FDTD results, the computer code provided in [9] is utilized. This computer code computes the diffraction coefficient for TM (soft) polarization using the equation

$$
\begin{aligned}
D_{s}\left(\rho, \phi, \phi^{\prime}, \omega\right) & \\
=\frac{-e^{-j \frac{\pi}{4}}}{2 n \sqrt{2 \pi \beta}}[ & \left(\cot \left(\frac{\pi+\xi^{-}}{2 n}\right) F\left(\beta, \rho, g^{+}\left(\xi^{-}\right)\right)\right. \\
& \left.+\cot \left(\frac{\pi-\xi^{-}}{2 n}\right) F\left(\beta, \rho, g^{-}\left(\xi^{-}\right)\right)\right) \\
& -\left(\cot \left(\frac{\pi+\xi^{+}}{2 n}\right) F\left(\beta, \rho, g^{+}\left(\xi^{+}\right)\right)\right. \\
& \left.\left.+\cot \left(\frac{\pi-\xi^{+}}{2 n}\right) F\left(\beta, \rho, g^{-}\left(\xi^{+}\right)\right)\right)\right]
\end{aligned}
$$

where $\xi^{+}=\phi+\phi^{\prime}, \xi^{-}=\phi-\phi^{\prime}, n$ is the wedge factor, which is 1.5 for a right-angle wedge, and $\mathrm{F}(\mathrm{X})$ is the Fresnel's transition 


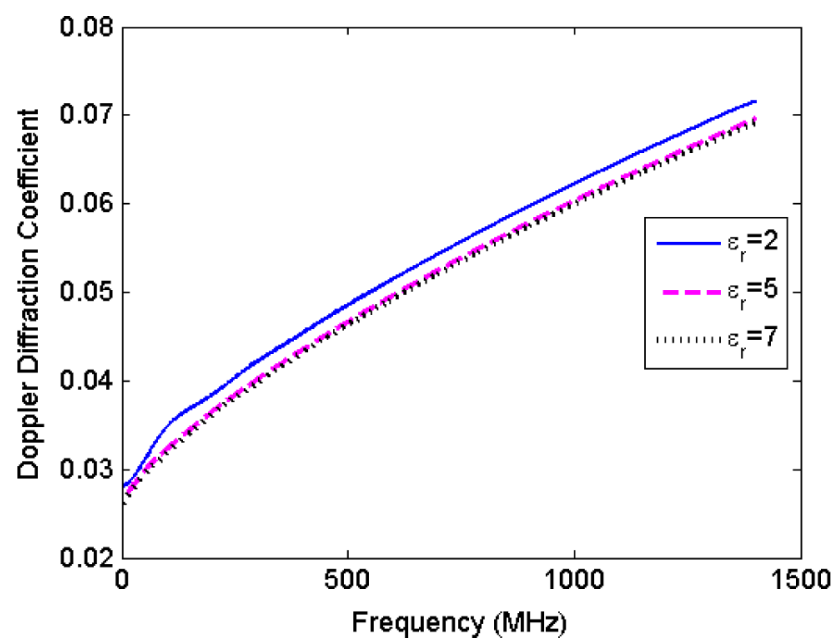

Fig. 4. Comparison between the Doppler diffraction coefficients for the lossless dielectric wedges of Fig. 3 vibrating horizontally, by $6 \mathrm{~mm}$ in the $+x$-direction under peak applied strain.

function computed by the asymptotic expressions for large and small arguments

$$
\begin{aligned}
& F(X) \approx\left(\sqrt{\pi X}-2 X e^{j \frac{\pi}{4}}-\frac{2}{3} X^{2} e^{-j \frac{\pi}{4}}\right) e^{j\left(\frac{\pi}{4}+X\right)} \\
& \begin{array}{l}
(\text { for } X<0.3) \\
F(X) \approx 1+j \frac{1}{2 X}-\frac{3}{4 X^{2}}-j \frac{15}{8 X^{3}}+\frac{75}{16 X^{4}} \\
\quad(\text { for } X>5.5) .
\end{array}
\end{aligned}
$$

As shown in Fig. 2, there is very good agreement between the analytical UTD results and the FDTD simulated results. Specifically, the relative error is less than $1 \%$ from $\sim 50$ to $1500 \mathrm{MHz}$.

\section{DifFRACTION COEFFICIENTS OF VIBRATING CONDUCTING AND DIELECTRIC WEDGES}

The model described in Section II-B is now employed to calculate the diffraction coefficients of vibrating wedges composed of different materials. For comparison, the diffraction coefficients of the unperturbed stationary wedges are also shown.

First, Fig. 3 shows the FDTD-calculated diffraction coefficient at Point $\mathrm{F}$ for three unperturbed dielectric right-angle wedges of relative permittivity 2,5 , and 7 . Fig. 4 then shows the FDTD-calculated Doppler diffraction coefficient at the same Point $\mathrm{F}$ for the same wedges vibrating $6 \mathrm{~mm}$ (two grid cells) horizontally to the right (in the $+x$-direction). To obtain the Doppler diffraction coefficient, the electric field time-waveform at Point F from the FDTD model having the unperturbed wedge is subtracted from that of the FDTD model having the wedge under peak applied strain (shifted $6 \mathrm{~mm}$ to the right), as

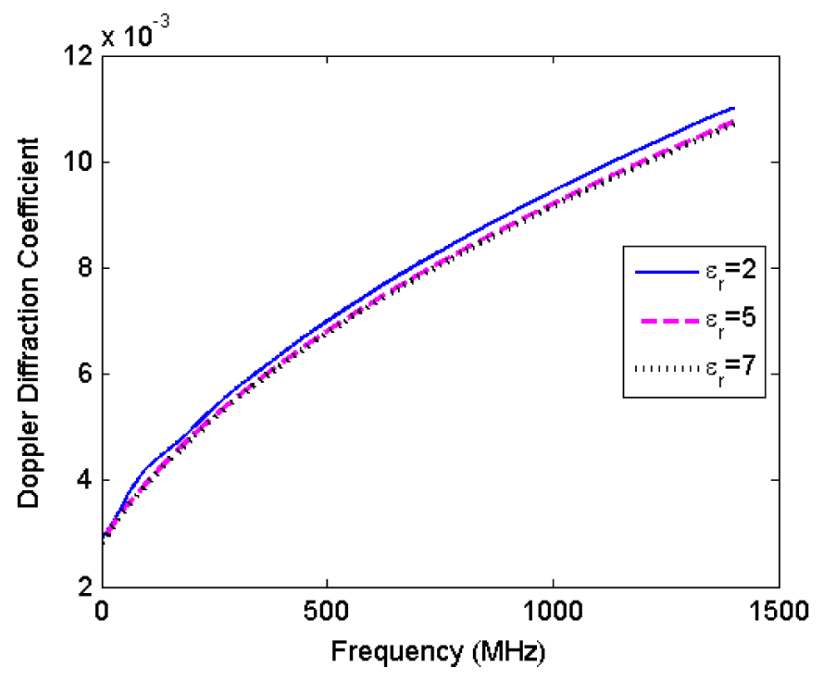

Fig. 5. Comparison between the Doppler diffraction coefficients for the lossless dielectric wedges of Fig. 3 vibrating vertically, by $6 \mathrm{~mm}$ in the $-y$-direction under peak applied strain.

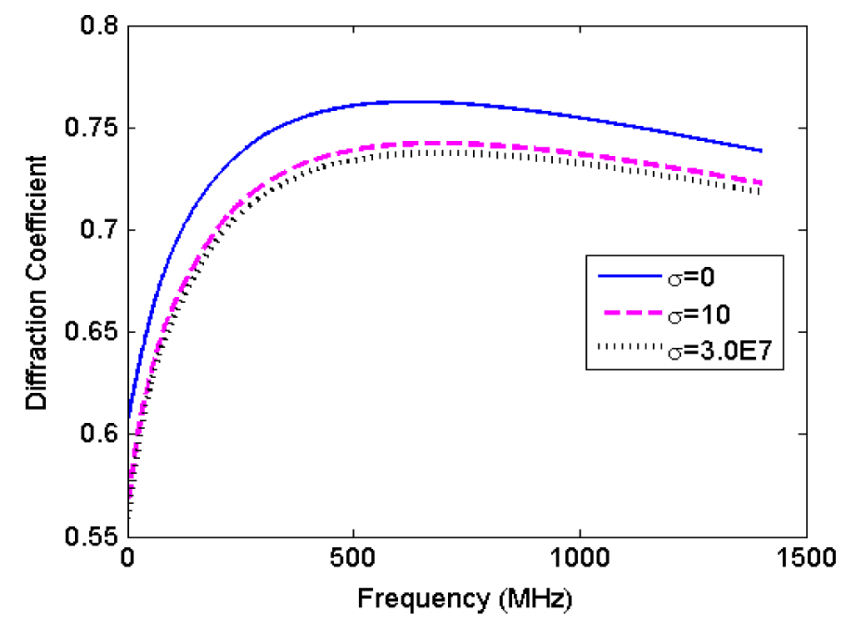

Fig. 6. Comparison between the diffraction coefficients for lossy, unperturbed dielectric right-angle wedges having a relative permittivity 7 and a conductivity 0,10 , and 3.0E7, respectively.

shown in (6) at the bottom of the page. The Doppler diffraction coefficient is then calculated as

$$
D_{\text {Doppler }}(\rho, \phi, \omega)=H_{\text {Doppler }}(\rho, \phi, \omega) \sqrt{r} e^{j \beta r} .
$$

Note in this case the surface of the wedge is not deformed. Under peak applied strain in this scenario, the entire right, straight side of the wedge is shifted to the right by $6 \mathrm{~mm}$. However, arbitrary deformations may be considered, either through higher resolution FDTD brute-force models or via lower resolution FDTD models employing the SBC method to resolve the vibrations.

$$
H_{\text {Doppler }}(\rho, \phi, \omega)=\frac{F\left\{X_{\text {dif, unpertured }}(\rho, \phi, t)-X_{\text {dif, peak applied strain }}(\rho, \phi, t)\right\}}{F\left\{X_{\text {inc }}(0, \phi, t)\right\}} .
$$




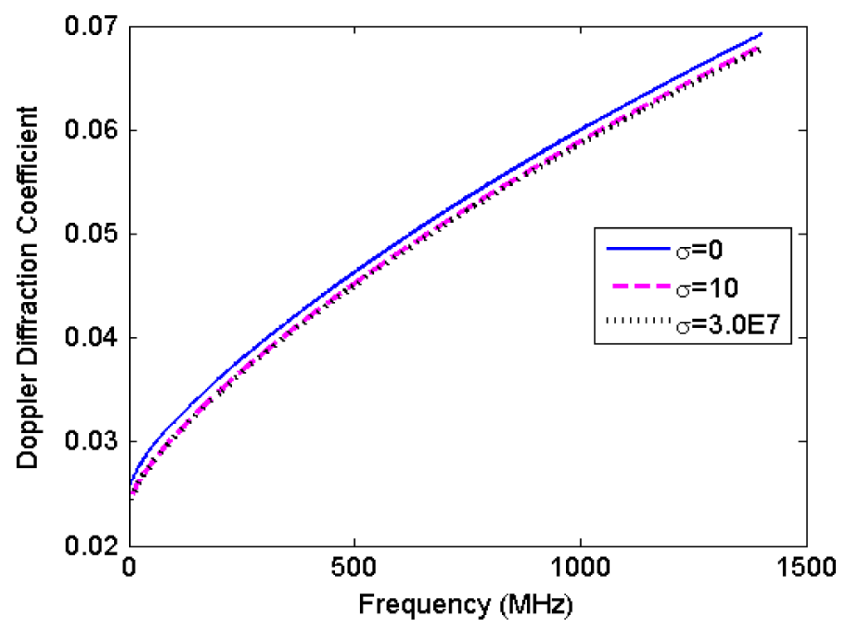

Fig. 7. Comparison between the Doppler diffraction coefficients for the lossy dielectric wedges of Fig. 6 vibrating horizontally, by $6 \mathrm{~mm}$ in the $+x$-direction under peak applied strain.

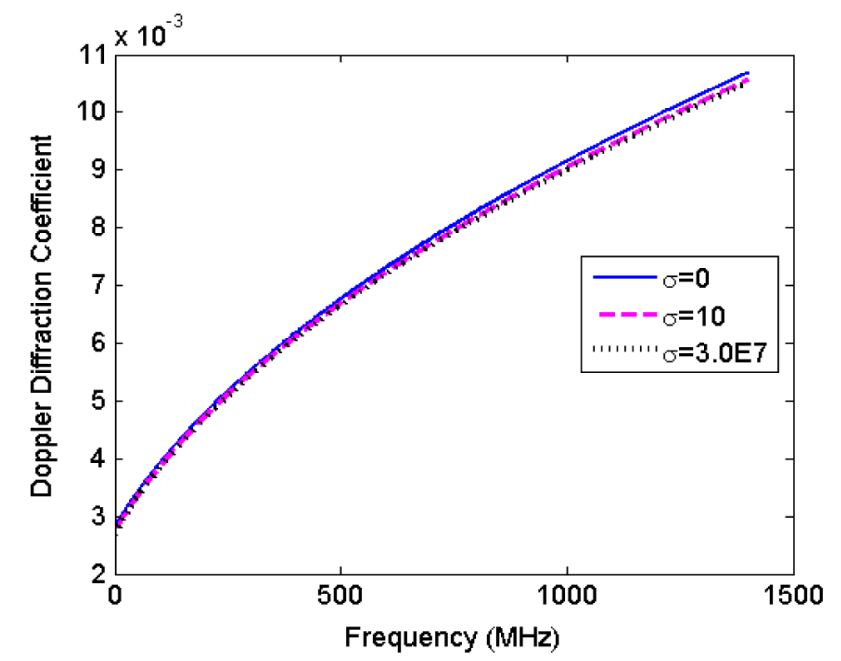

Fig. 8. Comparison between the Doppler diffraction coefficients for the lossy dielectric wedges of Fig. 6 vibrating vertically, by $6 \mathrm{~mm}$ in the $-y$-direction under peak applied strain.

We first notice by comparing the results of Figs. 3 and 4 that the amplitude of the Doppler diffraction coefficient is reduced by an order of magnitude relative to the stationary diffraction coefficient. Also, the amplitude of the Doppler diffraction coefficient increases with increasing frequency.

Fig. 5 shows the Doppler diffraction coefficient at Point $\mathrm{F}$ for the same wedges of Fig. 3, but this time vibrating $6 \mathrm{~mm}$ vertically downward (in the $-y$-direction). Comparing Fig. 5 to Fig. 4, we see that the Doppler diffraction coefficient is reduced by another order of magnitude when the wedge is vibrating downward versus to the right.

Next, Fig. 6 illustrates the FDTD-calculated diffraction coefficients at Point $\mathrm{F}$ for three unperturbed right-angle wedges of permittivity 7 and conductivity 0,10 , and 3.0E7, respectively.
Figs. 7 and 8 then illustrate the FDTD-calculated Doppler diffraction coefficient at Point F for the same wedges of Fig. 6 vibrating $6 \mathrm{~mm}$ horizontally to the right (in the $+x$-direction) and $6 \mathrm{~mm}$ vertically downward (in the $-y$-direction), respectively.

\section{CONCLUSION}

The diffraction coefficient of stationary and vibrating lossless and lossy dielectric wedges have been calculated via brute-force 2-D FDTD models. Depending on the direction of vibration, the Doppler diffraction coefficients are found to be between one and two orders of magnitude lower than the diffraction coefficient of the stationary wedge. Furthermore, the Doppler diffraction coefficient amplitude increases with increasing frequency over the spectrum of interest.

Although right-angle wedges are considered in this letter, note that FDTD modeling permits diffraction coefficient calculations for wedges and objects of arbitrary shapes and vibration characteristics. For instance, diffraction coefficients for a $45^{\circ}$ stationary wedge were calculated using FDTD in [10]. Also, the recently developed SBC algorithm provides an efficient means of modeling arbitrary vibration characteristics in FDTD models. For example, in [6] and [7], a variety of vibration modes of both square and circular objects are accounted for in the FDTD models using SBC.

\section{REFERENCES}

[1] G. Stratis, V. Anantha, and A. Taflove, "Numerical calculation of diffraction coefficients of generic conducting and dielectric wedges using FDTD," IEEE Trans. Antennas Propag., vol. 43, no. 10, pp. 1525-1529, Oct. 1997.

[2] K. S. Yee, "Numerical solution of initial boundary value problems involving Maxwell's equations in isotropic media," IEEE Trans. Antennas Propag., vol. AP-14, no. 3, pp. 302-307, May 1966.

[3] Taflove and S. C. Hagness, Computational Electrodynamics: The Finite-Difference Time-Domain Method, 3rd ed. Norwood, MA: Artech House, 2005.

[4] Q. Wang, M. M. Hayat, B. Santhanam, and T. Atwood, "Sar vibrometry using fractional fourier transform processing," in Proc. SPIE Radar Sensor Technol. XIII, K. I. Ranney and A. W. Doerry, Eds., 2009, vol. 7308, DOI: $10.1117 / 12.818370$.

[5] J. A. Roden and S. D. Gedney, "Convolutional PML (CPML): An efficient FDTD implementation of the CFS-PML for arbitrary media," Microw. Opt. Technol. Lett., vol. 27, pp. 334-339, 2000.

[6] A. Buerkle and K. Sarabandi, "Analysis of acousto-electromagnetic wave interaction using sheet boundary conditions and the finite-difference time-domain method," IEEE Trans. Antennas Propag., vol. 55, no. 7, pp. 1991-1998, Jul. 2007.

[7] A. Buerkle and K. Sarabandi, "Analysis of acousto-electromagnetic wave interaction using the finite-difference time-domain method," IEEE Trans. Antennas Propag., vol. 56, no. 8, pp. 2191-2199, Aug. 2008.

[8] M. Zhao, J. D. Shea, S. C. Hagness, D. W. van der Weide, B. D. Van Veen, and T. Varghese, "Numerical study of microwave scattering in breast tissue via coupled dielectric and elastic contrasts," IEEE Antennas Wireless Propag. Lett., vol. 7, pp. 247-250, 2008.

[9] C. A. Balanis, "Geometrical theory of diffraction," in Advanced Engineering Electromagnetics. New York: Wiley, 1989, ch. 13.

[10] V. Anantha and A. Taflove, "Efficient modeling of infinite scatterers using a generalized total-field/scattered-field FDTD boundary partially embedded within PML," IEEE Trans. Antennas Propag., vol. 50, no. 10, pp. 1337-1349, Oct. 2002. 